\title{
The On The Fly imaging technique
}

\author{
J. G. Mangum ${ }^{1}$, D. T. Emerson ${ }^{2}$, and E. W. Greisen ${ }^{3}$ \\ 1 National Radio Astronomy Observatory, 520 Edgemont Road, Charlottesville, VA 22903, USA \\ e-mail: jmangum@nrao.edu \\ 2 National Radio Astronomy Observatory, 949 North Cherry Avenue, Tucson, AZ 85721, USA \\ e-mail: demerson@nrao.edu \\ 3 National Radio Astronomy Observatory, PO Box O, 1003 Lopezville Road, Socorro, NM 87801, USA \\ e-mail: egreisen@nrao.edu
}

Received 8 May 2007 / Accepted 31 August 2007

\begin{abstract}
Aims. The On-The-Fly (OTF) imaging technique enables single-dish radio telescopes to construct images of small areas of the sky with greater efficiency and accuracy.

Methods. This paper describes the practical application of the OTF imaging technique. By way of example the implementation of the OTF imaging technique at the NRAO 12 Meter Telescope is described.

Results. Specific requirements for data sampling, image formation, and Doppler correction are discussed.
\end{abstract}

Key words. telescopes - methods: observational - radio continuum: general - radio lines: general

\section{Introduction}

In an effort to become scientifically more efficient, astronomical observatories have incorporated innovative observing techniques to increase the throughput of their telescopes. The OnThe-Fly (OTF) imaging technique is an innovation which has been adopted at a number of radio observatories over the past forty years in a variety of forms. In the 1960's and 1970's, radio observatories which operate at centimeter wavelengths implemented the "drift scanning" technique (sometimes referred to as "nodding") to acquire continuum imaging measurements (cf. Haslam et al. 1970). Drift scanned measurements are obtained by slowly slewing the telescope in elevation with the Earth's rotation providing the second dimension. In the 1980's, a number of radio telescopes added beam-switched continuum imaging capabilities. Beam-switched continuum observations are acquired by slewing the telescope in a two-dimensional raster pattern over a given region of sky while rapidly wobbling the telescope's subreflector to obtain differential measurements of the total power. The Emerson et al. (1979) [EKH] algorithm allowed the deconvolution of these beam-switched measurements into total power images. This powerful imaging capability added considerably to our ability to understand the centimeter- and millimeter-wave continuum structure of the interstellar medium. In the 1990s a number of radio observatories implemented both spectral line and continuum OTF imaging capabilities (cf. Mangum 1999; Mangum et al. 2000).

As with many innovations, this "new" OTF imaging technique was just an adaptation of an old observing technique. The observations which signaled the birth of radio astronomy, made by Karl Jansky in 1932, were actually OTF observations (see Janksy 1932; and Fig. 1). Jansky's 20.5 MHz synchrotron measurements of the galactic center represent the first astronomical OTF observations. In the following, we describe the adaptation of Jansky's imaging technique to total power images obtained using a single dish telescope.

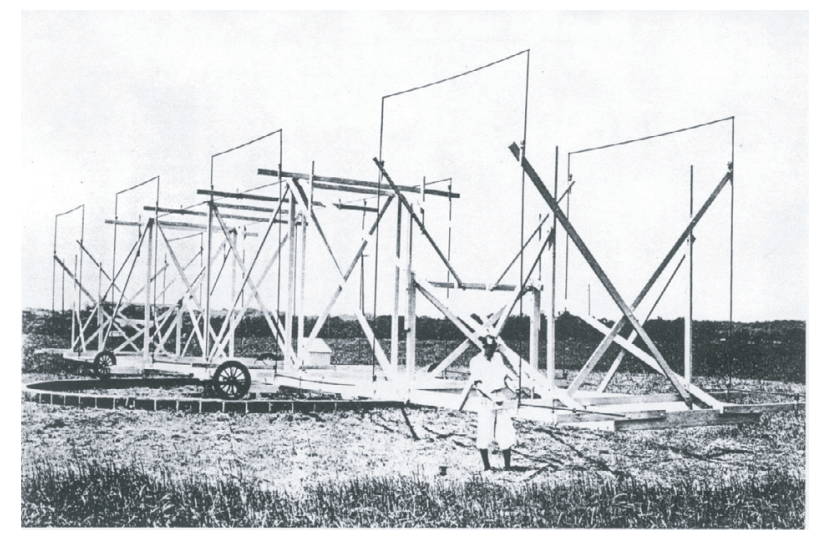

Fig. 1. Jansky and his telescope (Sullivan 1978).

\section{Advantages of OTF observing}

OTF mapping is an observing technique in which the telescope is driven smoothly and rapidly across a region of sky, or "field", while data and antenna position information are recorded continuously. This technique is in contrast to traditional mapping of discrete positions on the sky, which is sometimes called "stepand-integrate" or "point-and-shoot" mapping. The advantages to OTF mapping are:

1. telescope overhead is reduced significantly, since a specific position on the sky does not have to be acquired within a given tolerance (i.e. minimizing "dead-time");

2. the entire field is covered rapidly, minimizing changes in the properties of the atmosphere and the system, including antenna pointing and calibration. Systematic changes may occur from map to map, but such effects average down rapidly and may be correctable by cross correlation techniques. In general, global changes from map to map are more benign and easier to correct than drifts across a single map field; 


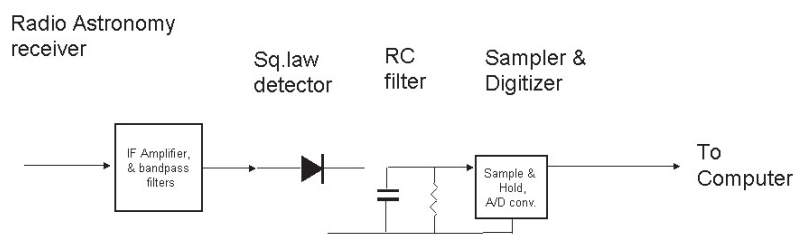

Fig. 2. Typical continuum detection scheme for a single dish telescope.

3. higher observing efficiency: the radiometer equation for switched measurements is given by the following

$\sigma=\frac{T_{\text {sys }}}{\eta_{\text {spec }} \sqrt{\Delta v t_{\text {on }}}}\left[1+\frac{t_{\text {on }}}{t_{\text {off }}}\right]^{\frac{1}{2}}$

where $\sigma$ is the rms noise in a total power measurement, $T_{\text {sys }}$ is the system noise temperature, $\Delta v$ is the spectral resolution of the measurement, and $\eta_{\text {spec }}$ is the spectrometer efficiency. The optimum duration of an OFF measurement for any switched measurement is given by (Ball 1976)

$t_{\text {off }}^{\text {optimal }}=\sqrt{N} t_{\text {on }}$

where $N$ is the number of $\mathrm{ON}$ measurements made per $\mathrm{OFF}$ measurement, such that $t_{\text {scan }}=t_{\text {off }}+N t_{\text {on }}$. Therefore, for optimal observing the rms noise in a measurement is given by

$\sigma=\frac{T_{\text {sys }}}{\eta_{\text {spec }} \sqrt{\Delta v t_{\text {scan }}}}\left[1+\frac{1}{\sqrt{N}}\right]$

where $t_{\text {scan }}$, as defined above, is the total ON plus OFF measurement time. For $N \geq 100, \sigma$ is approximately a factor of two smaller than it is for an equivalent position switched measurement, which leads to a factor of four improvement in observing time efficiency.

\section{Sampling}

\subsection{Detection and sampling: the spectrum of the detected noise and signal}

When conducting single dish imaging observations, it is important to keep in mind the following facts about sampling and aliasing in radio astronomical mapping data. If you want to represent the full resolution of the telescope, you have to sample the data often enough to represent all the spatial frequencies detected by the antenna. Figure 2 illustrates a typical continuum detection scheme of a single dish telescope; this is the simplest case, but the same principles apply to detection in a spectrometer, or even direct digitization at the IF and subsequent processing by software or firmware. In the example shown, the radio astronomical signal is amplified and bandpass filtered, before passing into a square law detector, possibly some simple filtering, and then into an Analog-to-Digital (A/D) converter. The A/D converter, or later processing, usually incorporates some signal integration before delivering the sampled data.

Figure 3 illustrates the low frequency spectrum of the signal and noise immediately after the square law detector and in the subsequent processing steps. With the antenna scanning at a constant speed over a point source, its response produces, after the detector, a time-varying signal whose spectrum corresponds to the spatial frequency response of the antenna. The horizontal axis of Fig. 3 could be spatial frequency, measured in units proportional to $\frac{d}{\lambda}$ ( $d$ is a baseline distance), or the frequency after the detector measured in Hz. Any antenna has a spatial frequency

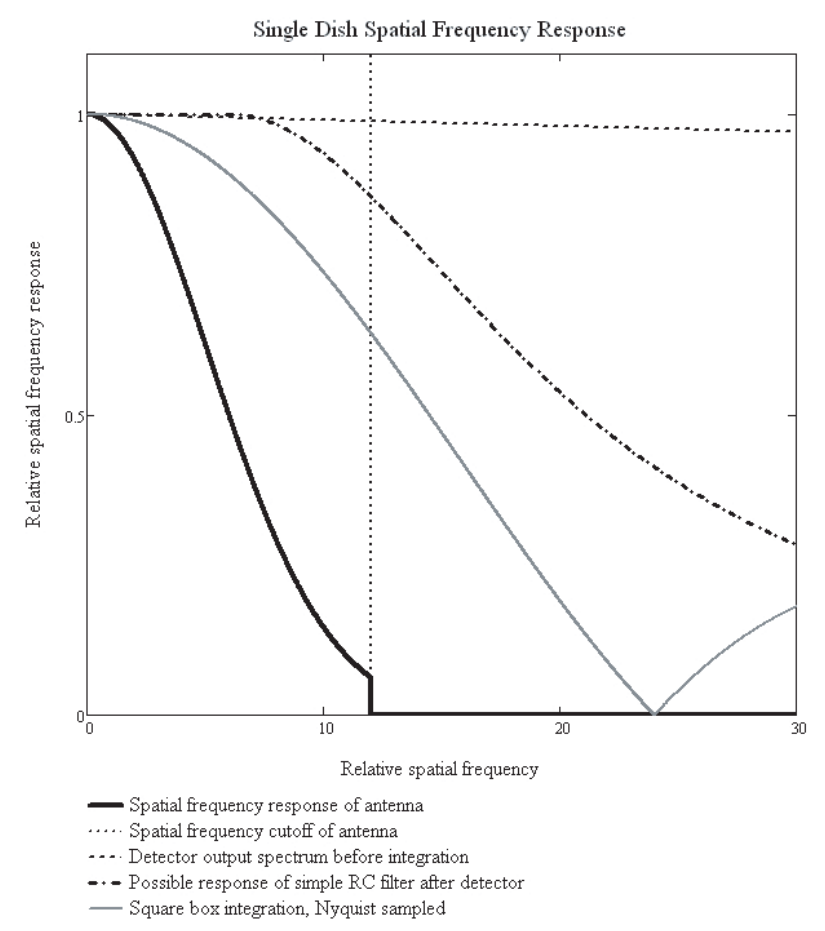

Fig. 3. Illustration of the low-frequency spectral response of the signal and noise after square law detection.

cut-off; with an antenna of diameter $D$ and scanning speed of $\Omega$ radians/second, after the detector the signal voltage will have a cut-off frequency of $\left(\frac{D}{\lambda}\right) \Omega$, now measured in Hz. This is illustrated by the bold curve in Fig. 3. The signal cut-off frequency is shown by the vertical dashed line.

Figure 3 also shows the spectrum of noise (the dashed line) immediately after the detector; this is strictly the autocorrelation function of the IF passband, which means in practice that within the narrow range of post-detection frequencies of interest, it is white noise, with a flat spectrum. It is normal to include a simple low pass filter immediately after the detector, shown symbolically by the RC time constant in Fig. 2. This might have a gentle frequency cut-off such as illustrated by the dash-dot line of Fig. 3.

The digitizer usually includes some time integration as part of its operation; the output samples might be sampled $\mathrm{S}$ times per second, but each sample is an integration of $\frac{1}{S}$ seconds of data. This integration is equivalent to convolving the time sequence of data with a square-box function - equivalent also to convolving the sky image along the scanning direction by the corresponding square-box function. The integration of $S$ seconds by this square-box translates into an average of $S * \Omega$ radians of angle along the sky scanning track. The time-domain spectral response, or equivalently the spatial frequency response, of this square-box integration is illustrated in Fig. 3 for the case where the signal is exactly Nyquist sampled (two independent samples per $\frac{\lambda}{D}$ interval on the sky).

The ideal signal processing and detection scheme might use a perfect square-edged anti-aliasing filter in place of the simple RC-filter illustrated in Fig. 2, followed by a sampler operating at least at twice the spatial frequency cut-off, with an infinitely narrow window in the sampler, thereby avoiding the loss of high frequencies caused by the normal square box integration. However, to avoid the complexities (and impossibility) of building a perfect anti-aliasing filter, very often in practice the square-box 
integration is retained, but with a substantial increase in the sample rate above the Nyquist value.

\subsection{The consequences of undersampling}

The spectrum of noise and of signal at the output of the detector of a total power system depends on the details of the electronic design, but a representative system is illustrated in Figs. 2 and 3.

With OTF observing, the astronomical sky is convolved with the telescope beamshape. The telescope acts also as a low pass filter, removing all astronomical components beyond the intrinsic angular resolution of the antenna. The telescope beam and telescope scanning speed together give a low pass filter, with the conversion from spatial frequency to temporal frequencies being given by

$R(f)=r(s f) * \Omega$,

with $R(f)$ being the temporal frequency response in $\mathrm{Hz}$ and $r(s f)$ the spatial frequency response measured in units of $\frac{d}{\lambda}$, with $\Omega$ the telescope scanning speed in $\mathrm{rad} / \mathrm{s}$. The respective responses are illustrated in Fig. 3.

\subsubsection{Aliasing}

The minimum sampling interval on the sky, for an antenna with maximum dimension $\mathrm{D}$, is at intervals of $\frac{\lambda}{2 D}$. Assume that one undersamples on the sky, rather than later in the data processing. Suppose you have a $10 \mathrm{~m}$ dish, but you only sample at $\lambda /(2 \times$ $8 \mathrm{~m})$ rather than the $\lambda /(2 \times 10 \mathrm{~m})$ that you should. This means that the spatial frequencies present from the dish baselines of $8 \mathrm{~m}$ to $10 \mathrm{~m}$ get reflected back into the spatial frequencies of $8 \mathrm{~m}$ down to $6 \mathrm{~m}$. Not only have spatial frequencies from the $8 \mathrm{~m}$ to $10 \mathrm{~m}$ baselines been lost, but valid spatial frequencies from baselines of $6 \mathrm{~m}$ to $8 \mathrm{~m}$ have been corrupted. You can't tell if structure in your map with a spatial wavelength of $\lambda / 7 \mathrm{~m}$ is genuine, or was really structure at $\lambda / 9 \mathrm{~m}$ which has been aliased on top of any genuine $\lambda / 7 \mathrm{~m}$ spatial wavelength signal. In this sense, undersampling the sky is really twice as bad as you might have thought.

Figures 4 and 5 show the spatial frequency response and aliased noise power for Nyquist sampling while using a square box sampling function, of width equal to the sampling interval. The power $P_{s}$ in the random noise fluctuations in frequencies up to the properly sampled spatial frequency, indicated by the vertical dashed line at 0.5 on the abscissa of Fig. 4 , is proportional to:

$P_{s}=\int_{0}^{0.5}\left(\frac{\sin (\pi x)}{\pi x}\right)^{2} \mathrm{~d} x$.

The power $A_{n}$ in the random fluctuations in frequencies beyond that frequency, in absence of any anti-aliasing or other filters, is proportional to:

$A_{n}=\int_{0.5}^{\infty}\left(\frac{\sin (\pi x)}{\pi x}\right)^{2} \mathrm{~d} x$.

This noise power $A_{n}$, which cannot contain any astronomical information, is then aliased back into the signal passband below 0.5 . The random (rms) noise voltage is then increased by the ratio:

$$
\sqrt{\frac{A_{n}+P_{s}}{P_{s}}}=1.137
$$

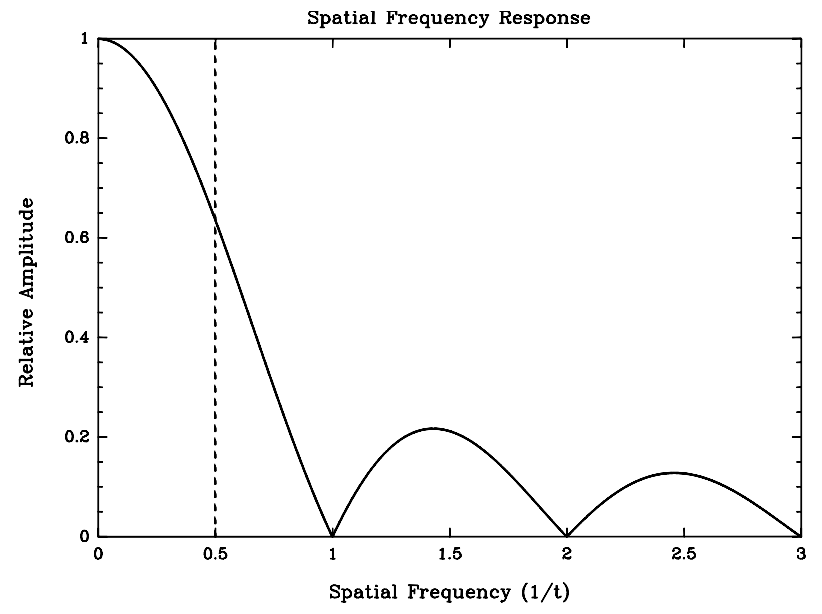

Fig. 4. Spatial frequency response for square box Nyquist sampling.

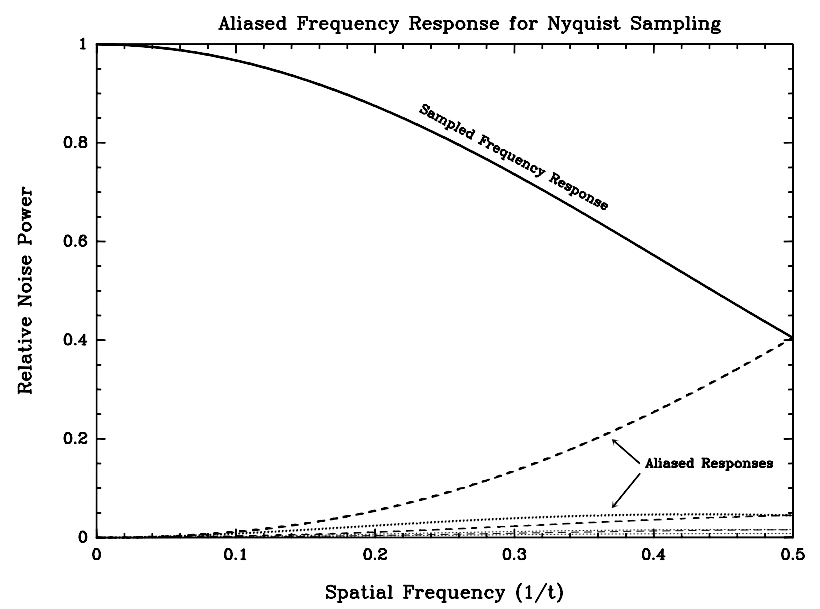

Fig. 5. Aliased noise power for square box Nyquist sampling in the absence of a separate anti-aliasing filter.

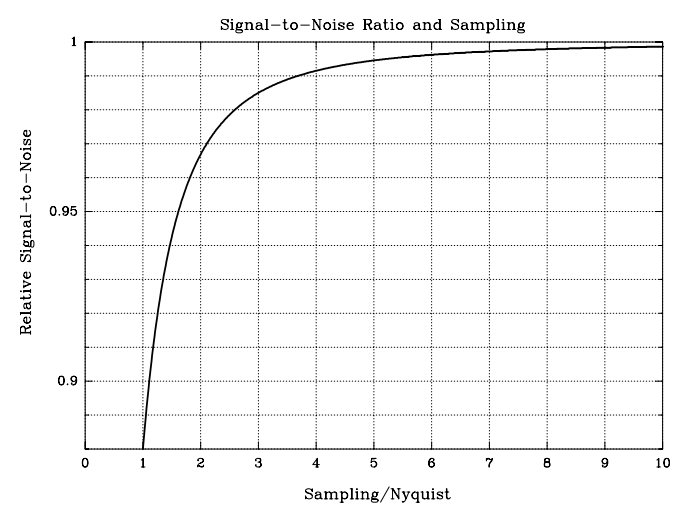

Fig. 6. Signal-to-noise degradation due to noise aliasing of an inadequately sampled image using square box sampling.

equivalent to a loss in signal-to-noise of $12 \%$. Therefore, the noise at frequencies to the right of 0.5 on the abscissa of Fig. 5, aliased back into the region of interest to the left of 0.5 , leads to an overall degradation in signal-to-noise of the image of $12 \%$.

The noise power degradation due to sampling is a function of the level of oversampling in the image plane. Figure 6 shows the amount of signal-to-noise degradation one can expect due to noise aliasing of an inadequately sampled signal using square box sampling. 
How important undersampling is depends on exactly what the illumination taper is, how important it is that you retain the maximum possible resolution of the telescope, how good a dynamic range you want in the observations, and at some level how much fine scale structure there is in the source itself. If you only sample at 0.833 Nyquist (e.g. $F W H M / 2$ rather than $F W H M / 2.4$ for a $-13 \mathrm{~dB}$ illumination taper), what matters is the energy in the data at spatial wavelengths shorter than $\lambda /(2 \times 0.8 \times D)$. So in a sense you need to ask what the illumination taper is at a radius of $0.4 \times D$ on the dish surface. The spatial frequency response of a single dish is the autocorrelation function of the voltage illumination pattern. So, you need to calculate how much area there is under the 2-D autocorrelation function beyond spatial frequencies of $0.8 \times D$, compared to the area within $0.8 \times D$. This ratio is some measure of the dynamic range. A better definition of dynamic range might take into account the spatial frequency structure of the source. If the source has no structure on scales smaller than $\lambda /(2 \times 0.8 \times D)$, then you don't need to sample at the full $\lambda /(2 \times D)$ anyway.

There are circumstances where it is perfectly rigorous to undersample the data in the absence of a separate anti-aliasing filter. For example, if you have a $10 \mathrm{~m}$ dish, and you are taking data to compare with other observations that were made using a $1 \mathrm{~m}$ dish at the same wavelength (or the equivalent number of wavelengths at some other frequency) then you only need to sample the data at $\lambda /(2 \times 5.5 \mathrm{~m})$ or $\lambda / 11 \mathrm{~m}$. This is so because, when sampling a $10 \mathrm{~m}$ dish as if it were a $5.5 \mathrm{~m}$ dish, the spatial frequency components from baselines of $5.5 \mathrm{~m}$ out to $10 \mathrm{~m}$ will be reflected back into the data as if corresponding to baselines of $5.5 \mathrm{~m}$ down to $1 \mathrm{~m}$. So, the spatial frequency terms of the $1 \mathrm{~m}$ baseline and below will not have been corrupted. The data analysis of this undersampled data would apply a spatial frequency cutoff at $1 \mathrm{~m}$, and there will have been no corruption in this smoothed data caused by the undersampling. Putting it in more general terms, if you are going to be smoothing observations from a dish of diameter $D$ to simulate observations made with a smaller dish of diameter $d$, then the sampling interval only needs to be $\lambda /(d+D)$ rather than $\lambda /(2 \times D)$.

\subsubsection{Beam broadening}

Since the telescope beam is effectively convolved with a squarebox function equal to the distance the telescope moves in one sample period, there is a degradation of spatial resolution due to the data acquisition process. If you set an arbitrary criterion that the telescope beam shouldn't be broadened by more than $1 \%$, then you need to sample at least 4.5 points per FWHM beam width. Note that since Nyquist sampling is about 2.4 points per $F W H M$, assuming an illumination taper of about $-13 \mathrm{~dB}$; for a uniformly illuminated circular aperture the factor would be about 2.06, while for a $-20 \mathrm{~dB}$ taper it becomes 2.60. This means that one needs to sample at about twice Nyquist to avoid degradation of resolution worse than $1 \%$.

\subsubsection{Sampling summary}

1. To avoid both beam degradation and loss of signal/noise ratio, one must sample at least twice Nyquist, or about 5 points per $F W H M$.

2. With few exceptions, undersampling at data acquisition is not recommended.

3. There are other aspects that make it desirable to sample more often than the Nyquist rate, as is often recommended for

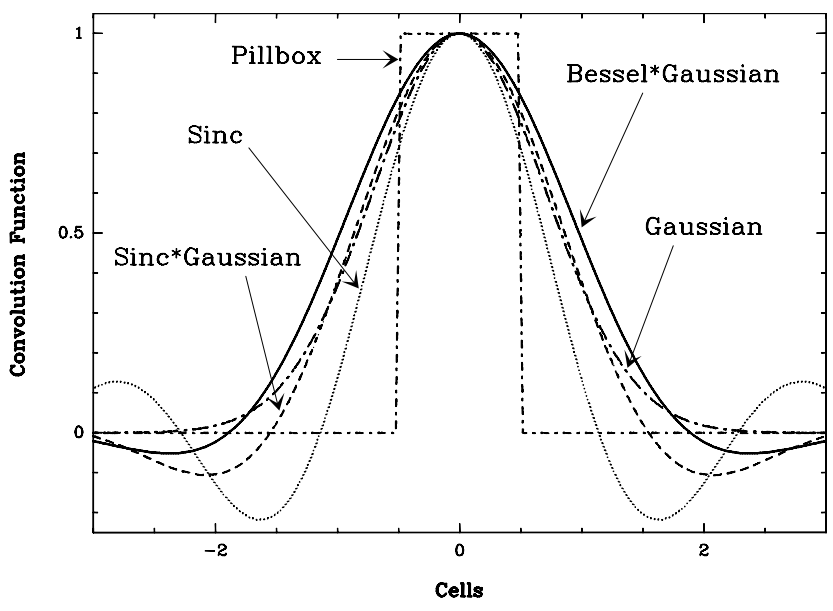

Fig. 7. Representative convolution functions.

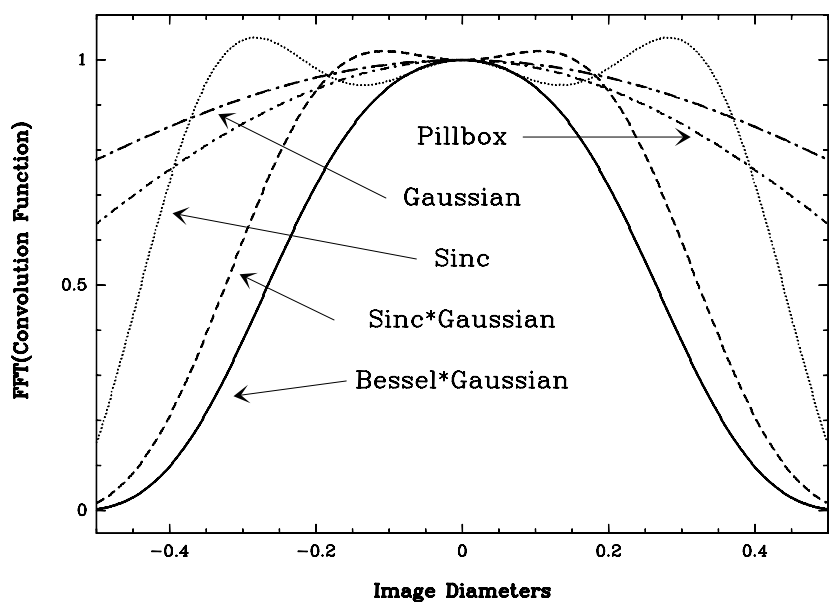

Fig. 8. Representative Fourier transforms of convolution functions.

OTF observing at many millimeter-wave telescopes. These are practical points like how well gridding or interpolation works with a finite sized gridding or interpolation function. For example, a little oversampling may enable you to reduce the convolution (interpolation) function by a factor of a few, saving a huge amount of computational overhead at the expense of a few per cent more data.

\section{Gridding (convolution)}

The final image is constructed by smoothing the data at whatever coordinates they were observed and then re-sampling them on a regular image grid. The smoothing is, in fact, an interpolation, which can include weighting and averaging, rather than a strict convolution. However, when the data are sampled in a dense and nearly uniform fashion, the interpolation approximates a convolution. Figure 7 shows some representative convolving functions which may be used in the data gridding. The effect of the convolution is best seen by examining its impact, a multiplication, in the Fourier, spatial frequency, space. The Fourier transforms of the convolving functions are illustrated in Fig. 8.

The spatial frequency response pattern of the single dish is effectively multiplied by the Fourier transform of the convolving function. If the function were to have no effect on the data, then its Fourier transform should have value 1.0 out to the maximum spatial frequency of the telescope and 0.0 outside that radius. This function is, in one dimension, a sinc function, 
Table 1. Convolution function factor.

\begin{tabular}{|c|c|c|c|c|}
\hline Function & Parms & $\begin{array}{r}\text { Functi } \\
\eta \text { Linear }\end{array}$ & $\begin{array}{l}\text { n Form } \\
\eta \text { Circular }\end{array}$ & $R_{\text {support }}$ \\
\hline Pill Box & $\ldots$ & 1.00 & 0.78 & 0.5 \\
\hline $\exp \left[-\left(\frac{|z|}{b}\right)^{2}\right]$ & $b=1.00$ & 3.14 & 3.14 & 3.0 \\
\hline$\frac{\sin \left(\frac{\pi|k|}{a}\right)}{\frac{\pi[\pi}{a}}$ & $a=1.14$ & 1.36 & 1.16 & 3.0 \\
\hline$\frac{\sin \left(\frac{\pi|z|}{a}\right)}{\frac{\pi|z|}{a}} \times \exp \left[-\left(\frac{|z|}{b}\right)^{2}\right.$ & $a=1.55, b=2.52$ & 2.33 & 1.43 & 3.0 \\
\hline$\frac{J_{1}\left(\frac{a \mid l}{c}\right)}{\frac{z}{c}} \times \exp \left[-\left(\frac{|z|}{b}\right)^{2}\right]$ & $b=2.52, c=1.55$ & 3.59 & 3.03 & 3.0 \\
\hline
\end{tabular}

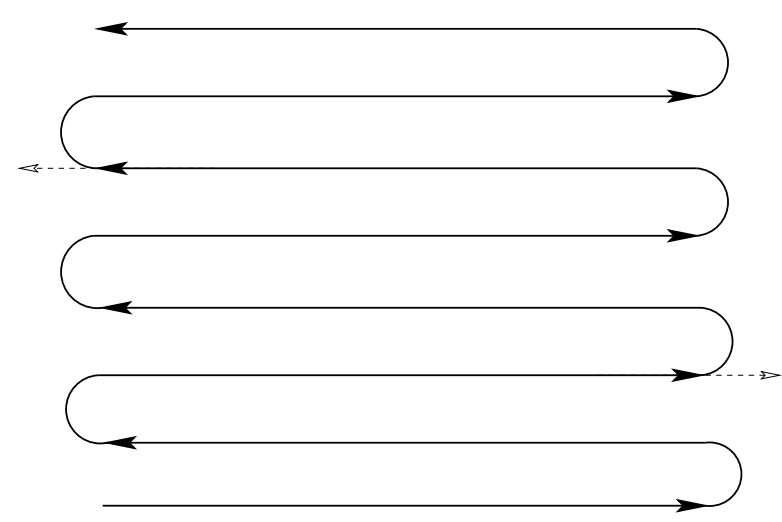

Fig. 9. An example of a raster scanning pattern. In this example, three scanning rows are acquired per reference ("off") measurement.

$\sin (\pi x / N S) /(\pi x / N S)$ and, in two dimensions, a Bessel function $J_{1}(\pi x / N S) /(\pi x / N S)$. Unfortunately, both of these functions die off very slowly, making the convolution operation excessively expensive. A compromise function is obtained by tapering the convolution function by a fairly narrow Gaussian, allowing the function to be truncated outside a restricted region. In the Fourier space, this has the effect of reducing the response to the largest spatial frequencies available to the telescope. These spatial frequencies are probably already tapered by the response pattern of the feed horn, which is usually designed to avoid sensitivity to ground spillover.

Since we have a circular telescope and are gridding the image in two dimensions simultaneously, we use the circularly symmetric version of the convolving function, $J_{1}(r / a) /(r / a) \exp \left(-(r / b)^{c}\right)$. Optimizations of this function give the default values for $a, b$, and $c$ of $a=1.55$ (single-dishbeam-size / 3), $b=2.52$ (single-dish-beam-size / 3), $c=2$ (see Table 1), and a support radius equal to the single-dish-beam-size (FWHM) (Schwab 1980).

It is important to choose the sampling of the output image, and the width and support of the convolving function to avoid aliasing. This topic was discussed at length in Sect. 3.2.1. A suitable cell size would be less than or equal to one-third of the single-dish-beam-size.

Other convolving functions have various undesirable effects. For example, a sinc ${ }^{2}$ has a triangular Fourier transform. All non-zero spatial frequencies are weighted down linearly with frequency, degrading the telescope resolution. All other positive-only functions have a similar effect. One can construct convolving functions such as $\sin (x / a) \sin (y / a) /(x / a) /(y / a) \exp \left(-\left((x / b)^{2}+(y / b)^{2}\right)\right)$ which will actually improve the spatial resolution of the image over that of the single-dish beam. They do this at the cost of down-weighting

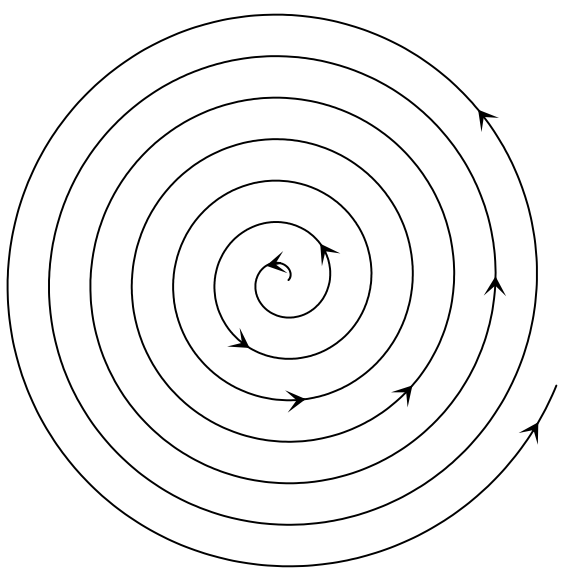

Fig. 10. An example of the spiral scanning pattern.

low spatial frequencies and thereby reducing the signal-to-noise of the observation (Greisen 1998).

\section{Scanning geometry}

The scanning geometry used to acquire an OTF map is usually dependent upon the type of source you are imaging and the ability to command the antenna to execute complex patterns. Scanning geometries that have been implemented at existing radio telescopes include:

Raster: usually the first scanning geometry implemented at a telescope as it is the simplest to implement within a telescope monitor and control system. Figure 9 is a pictorial display of this scanning geometry.

Spiral: an efficient scanning geometry when imaging a circular or elliptical source (see Fig. 10).

Hypocycloid: for imaging a non-circular source which is large in relation to the resolution element of your observations, the hypocycloid is a very motion-efficient pattern (i.e. no antenna motion efficiency losses due to turn-around; see Fig. 11).

\section{OTF imaging in practice}

It is often important to estimate the amount of integration time and the rms noise level an OTF image will have based on the observing parameters. In the following, we derive these quantities for a raster scanned OTF map. We also discuss an often overlooked aspect of wide-field spectroscopy involving the doppler correction.

\subsection{OTF map parameters}

In the following we describe some of the OTF map parameters that one must generally calculate in order to setup an OTF observation.

\subsubsection{Row sampling rate}

It is very important that you set up your map to be properly sampled in all coordinates. If you under sample, you will miss information in the image field, you will be unable to combine your image accurately with that from interferometers or other single-dish telescopes, and you may introduce artifacts from the 


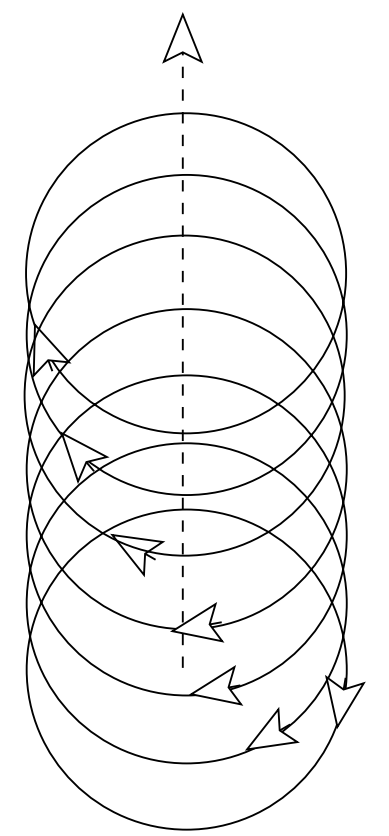

Fig. 11. An example of the hypocycloid scanning pattern.

analysis algorithms (see Sect. 3.2.1). Keep in mind that you can always smooth the map after it is taken to degrade the resolution and improve signal-to-noise.

The scanning rows must be spaced at no more than the Nyquist spacing, which is also known as "critical sampling" and is given by

$$
\begin{aligned}
\theta_{\mathrm{N}} & \equiv \frac{\lambda_{\mathrm{obs}}}{2 D} \text { radians } \\
& \simeq \frac{30918}{D(m) v_{\mathrm{obs}}(\mathrm{GHz})} \operatorname{arcsec} .
\end{aligned}
$$

In practice, though, a small amount of oversampling is recommended. If the rows are critically spaced, small scanning errors can result in the map being under sampled. In addition, the tail of the gridding function used by the analysis procedure extends slightly beyond the information cutoff of the telescope, which will result in some noise being aliased into each grid cell (see Sect. 3.2.1). A reasonable approach is to sample both sky coordinates by:

$$
\begin{aligned}
\theta_{\text {row }} & =\left(\frac{\lambda_{\mathrm{obs}}}{2 D}\right) \times 0.9-\delta \\
& \simeq\left(\frac{27826}{D(m) v_{\mathrm{obs}}(\mathrm{GHz})}\right)-\delta \operatorname{arcsec}
\end{aligned}
$$

where the factor of 0.9 is an oversampling factor and the factor of $\delta$ is a guard band to accommodate any scanning errors. For the NRAO $12 \mathrm{M}$ Telescope $\delta=2$ was used. Figure 12 is a plot of this relationship assuming a $12 \mathrm{~m}$ aperture.

\subsubsection{Scanning rate}

Map sampling along the scanning direction is dependent upon the integration (or "dump") time of the data acquisition system (i.e. spectrometer) being used. Since the spectrometers must integrate for a finite interval before being dumped, the data are "square-box" binned. To avoid noise aliasing and beamsmearing problems due to this binning (see Sect. 3.2.1), one

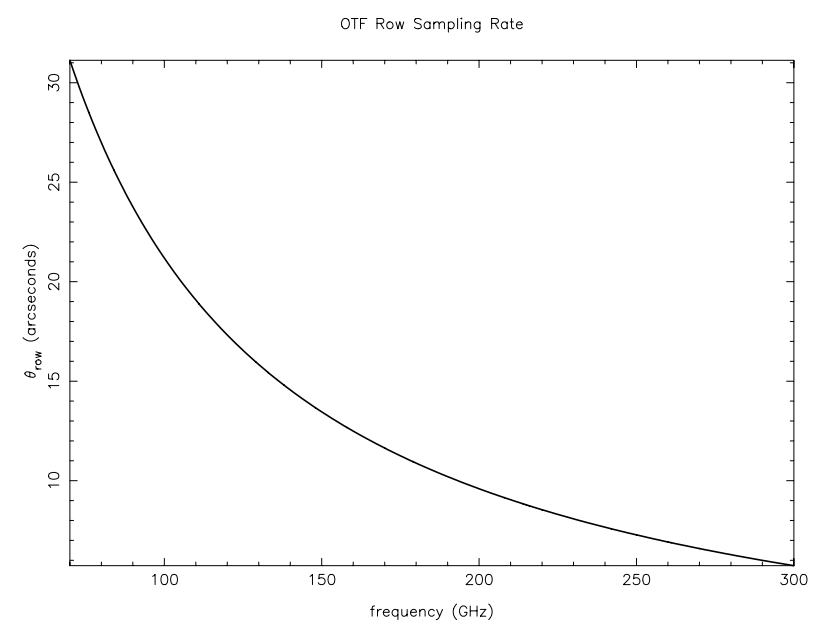

Fig. 12. $\theta_{\text {row }}$ as a function of observing frequency for a $12 \mathrm{~m}$ aperture.

needs to oversample in the scanning direction. The scan rate may be written as

$R(\operatorname{arcsec} / \mathrm{s})=\frac{\theta_{\mathrm{N}}}{n_{\mathrm{os}} t_{\mathrm{dump}}}$

where $\theta_{\mathrm{N}}$ is the Nyquist spacing (Eq. (8)), $n_{\mathrm{os}}$ is an oversampling factor, and $t_{\text {dump }}$ is the spectrometer data dumping interval. At the NRAO 12 Meter Telescope, $t_{\text {dump }}=0.1$ seconds for spectral line OTF, $0.25 \mathrm{~s}$ for continuum OTF. The general recommendation to observers when using the $12 \mathrm{~m}$ system was to use a minimum value for $n_{\text {os }}$ of 2 , which will result in $<3 \%$ increased noise as a result of aliasing and minimal beam broadening (see Sect. 3.2.2). Note that it is quite acceptable to oversample by much larger factors than the minimum value suggested above, particularly in the scanning direction. There are some tradeoffs to be considered, however:

1. large factors of oversampling will produce better signal-tonoise on a single coverage of the field and may result in simpler data processing, i.e. fewer maps to average together;

2 . on the other hand, the longer a single coverage takes, the more susceptible your image will be to drifts in the system, such as pointing and atmosphere. This eliminates some of the advantages of fast mapping mentioned above.

A good compromise is to use a row spacing computed from Eq. (9) and a scan rate calculated from Eq. (10), with $n_{\mathrm{OS}}>2$. Other circumstances may come into play, of course. For example, if the field size is very small, you may choose to slow the scan rate (increase $n_{\mathrm{os}}$ ) substantially.

\subsubsection{Map rms}

When planning an OTF observing run you must calculate how much integration time you need for each sampling cell to reach the desired signal-to-noise level. First determine the integration time required using the standard Radiometer Equation (Eq. (1))

$\sigma_{\text {cell }}=\frac{T_{\text {sys }}}{\eta_{\text {spec }} \sqrt{\Delta v t_{\text {cell }}}}\left[1+\frac{t_{\text {cell }}}{t_{\text {off }}}\right]^{\frac{1}{2}}$,

where $T_{\text {sys }}$ is the system temperature, $\eta_{\text {spec }}$ is the spectrometer efficiency (typically $\simeq 1$ ), $\Delta v$ is the spectral resolution, $t_{\text {cell }}$ is the time spent integrating on a cell, and $t_{\text {off }}$ is the off-source integration time. For spectral line OTF, the time spent integrating on a 
given cell is typically one second or less, while the off-source integration time is often several seconds ( 10 seconds was the default value for the NRAO $12 \mathrm{~m}$ Telescope). Therefore, $t_{\text {cell }} \ll t_{\text {off }}$ and we can rewrite Eq. (11) as

$t_{\text {cell }}=\frac{1}{\Delta v}\left(\frac{T_{\text {sys }}}{\sigma_{\text {cell }}}\right)^{2}$.

The integration time in a map cell is a function of the scanning rate and the number of coverages of the map field, and should be calculated with respect to the time accumulated in a Nyquist sampling cell. For illustration we assume a raster scanning geometry. If we define $\theta_{x}$ to be the angular distance along the scanning row, $\theta_{\text {ramp }}$ to be a "ramp-up" distance at the beginning and end of each row, and $R$ to be the scanning rate (see Sect. 6.1.2), then the time to scan one row is

$t_{\text {row }}=\frac{\theta_{x}+2 \theta_{\text {ramp }}}{R}$.

If we also define $\theta_{y}$ to be the angular height of the map and $\theta_{\text {row }}$ to be the angular sampling interval between scanning rows, then the number of rows in the map is given by

$N_{\text {row }}=\frac{\theta_{y}}{\theta_{\text {row }}}$.

The number of independent sampling cells in the map is

$N_{\text {cell }}=\frac{\left(\theta_{x}+2 \theta_{\text {ramp }}\right) \times \theta_{y}}{\theta_{\mathrm{N}}^{2}}$

where we are approximating the cells as rectangular. The integration time per cell, $t_{\text {cell }}$, is related to the above three quantities as follows

$t_{\text {cell }}=\frac{t_{\text {row }} N_{\text {row }}}{N_{\text {cell }}} \times \eta$

where $\eta$ is a convolution function correction factor which takes account of the fact that we are using a tapered function (a Gaussian-tapered Bessel function) instead of a pure sinc. We define the convolution function $C(u)_{m}$ as follows:

$C(u)_{m}=\int_{0}^{\infty} C(u) \mathrm{d} u$.

The $\eta$ correction factor is then given by

$\eta \equiv \frac{C(u)_{m}}{C(0)_{m}}$

for the truncated functions used in the AIPS task SDGRD (see Sect. 4 and Table 1). Combining Eqs. (13)-(16), and (18), we find that

$t_{\text {cell }}=\frac{3.03 \theta_{\mathrm{N}}^{2}}{R \theta_{\text {row }}}$.

Combining this relation for $t_{\text {cell }}$ with our Radiometer Equation (Eq. (11)), we find that the rms noise per cell in an OTF map is given by

$\sigma_{\text {cell }}=\frac{T_{\text {sys }}}{\theta_{\mathrm{N}}}\left(\frac{R \theta_{\text {row }}}{3.03 \Delta v}\right)^{\frac{1}{2}}$.

Note that a given rms noise per cell obtainable with multiple coverages and/or polarizations is given by

$\sigma=\frac{\sigma_{\text {cell }}}{\sqrt{N_{\mathrm{c}}}}$ where $N_{\mathrm{c}}$ is the number of coverages and/or polarizations you combine to produce your final image. Note that you can also improve your map rms by spatially smoothing it after gridding (see Sect. 4).

\subsubsection{Total map time}

Again assuming a raster scanning geometry, the total time required to acquire a map must include not only the integration time scanning across the field but also the time for calibration and OFF integrations. This can be written as

$t_{\text {tot }}=N_{\text {row }}\left[t_{\text {row }}+\frac{t_{\text {off }}}{N_{\text {rpo }}}+\frac{t_{\text {cal }}}{N_{\text {rpo }} N_{\text {opc }}}+\frac{\epsilon}{N_{\text {rpo }}}\right]$

where $N_{\text {row }}$ is the number of rows in the map, $t_{\text {row }}$ is given by Eq. (13) above, $t_{\text {off }}$ is the OFF integration time, $t_{\text {cal }}$ is the calibration integration time (i.e. amplitude calibration and sky sample times), $N_{\text {rpo }}$ is the number of rows per OFF measurement you do, $N_{\mathrm{opc}}$ is the number of OFF measurements per calibration measurement (amplitude calibration and sky) you make, and $\epsilon$ is the "overhead" time, which is the time that the telescope spends doing things other than integrating (like moving from OFF positions to the map field). The value for $\epsilon$ often depends mainly on how far away the OFF position is from the map, but $10 \mathrm{~s}$ is probably a good average estimate for vintage- $1980 \mathrm{~mm}$ radio telescopes. Most of the time the amplitude calibration measurement is taken at the same position and just before the OFF measurement, and so doesn't involve additional overhead. There may be other small overhead losses in moving from row to row and starting scans, but these losses can usually be neglected.

\subsection{Integration time and rms noise}

Based on the following definitions:

$\eta \equiv$ Convolution function factor. This is a measure of how much the data oversamples the convolution function.

$t_{\text {row }} \equiv$ Time to scan one row in the image.

$N_{\text {row }} \equiv$ Number of rows in image.

$\theta_{\text {row }} \equiv$ Row separation.

$R \equiv$ Scanning rate.

$N_{\text {cell }} \equiv$ Number of independent sampling intervals in image.

$\theta_{\text {Nyquist }} \equiv$ Nyquist sampling interval $(=\lambda /(2 D))$.

$T_{\text {sys }} \equiv$ System temperature.

$\Delta v \equiv$ Spectral resolution.

We can write the relation for the integration time per sampling interval as:

$$
\begin{aligned}
t_{\text {cell }} & =\frac{\eta t_{\text {row }} N_{\text {row }}}{N_{\text {cell }}} \\
& =\frac{\eta \theta_{\text {Nyquist }}^{2}}{R \theta_{\text {row }}}
\end{aligned}
$$

and the rms noise per sampling interval is:

$\sigma_{\text {cell }}=\frac{T_{\text {sys }}}{\theta_{N}}\left(\frac{R \theta_{\text {row }}}{\eta \Delta v}\right)^{\frac{1}{2}}$.

The factor $\eta$ is dependent upon the convolution function used to grid the OTF data. The $\eta$ factors for the convolution functions available in the AIPS single dish analysis programs are listed in Table 1. 


\subsection{Differential doppler correction}

Since the correction for the radial motions of the local standard of rest relative to a given source vary as a function of position on the sky, time, and telescope location, the conversion from spectral frequency to velocity varies over a given image. This is a subtle effect, traditionally ignored in spectral-line imaging. However, for a coordinate $1.4^{\circ}$ from the reference (e.g. a $2^{\circ}$ square field with a center reference), the error can be as large as $1.16 \mathrm{~km} \mathrm{~s}^{-1}$ (LSR velocities) or $0.79 \mathrm{~km} \mathrm{~s}^{-1}$ (heliocentric) when observed with the NRAO 12 Meter Telescope. The LSR velocity error changes significantly with time of year. Narrow-band observations of wide fields, such as observations of cold molecular regions, may be seriously affected by this effect. Fortunately, so long as the spectra are fully sampled in frequency, this effect can be fully corrected by doing the LSR calculations separately for every point of each map, for all dates, and then interpolating to a common frame and date in frequency space.

\subsection{Smoothing and signal-to-noise for critically-sampled data}

When you grid a critically-sampled (Nyquist-sampled) OTF map with a sensible convolution function (such as the Gaussiantapered Bessel function described in Sect. 4), the resulting spatial resolution is only minimally degraded. However, the noise spectrum of your gridded map is flat (or, equivalently, the noise in each gridded point is independent), while the astronomy spectrum follows that of the spatial frequency response of the antenna. If you now smooth the data significantly - say to a resultant beamwidth several times greater than the original spatial resolution - both the astronomical signal and the noise get smoothed. The effective increase in integration time is given roughly by $\frac{\text { new convolution function area }}{\text { original sampling cell area }}$ Obviously the signal-to-noise ratio increases by the square root of this quantity. Note that it is the ratio of the new convolution function to the original sampling that counts. This is because the high frequency terms of the original noise, which probably dominate the total noise, are reduced in amplitude much more than the astronomical signal, whose high-frequency components were already weighted down.

After you have smoothed the data once, the noise and astronomical signal now have a very similar spectrum. If you smooth the data a second time, again to a beamwidth several times larger than the current (smoothed) beamwidth, the effective integration time now increases roughly by $\frac{\text { new beam area }}{\text { current beam area }}$. It is now the ratio of the beams, independent of the sampling of either measurement. This in a sense is because the noise in adjacent samples of the smoothed data is no longer independent, so you don't gain as much in the second smoothing as was gained in the first.

The conclusion is that to be rigorous you need to know the noise spectrum as well as the data beamwidth in order to calculate what the effective integration time becomes when you smooth critically-sampled data. It depends on whether you can consider noise in adjacent samples independent. After the initial gridding of a critically-sampled data set, the noise is independent in adjacent samples. After you have smoothed the data once, the noise is no longer independent.

\section{OTF observing at the NRAO 12 meter telescope}

Before starting a spectral line or continuum OTF map at the 12 Meter, the telescope control system was configured to raster map the target field. For spectral line OTF, the map was taken in a total power observing mode in the sense that you acquired a calibration spectrum (a vane calibration) and a total power off measurement, followed by one or more total power scanning rows, typically made up of hundreds of individual spectra (the on measurements). For continuum OTF, the map was acquired using the continuum beam-switched observing mode. In this mode, the subreflector is switched between two positions (the "+" and "-" beam) in azimuth while the telescope scans. Each of the individual spectra or continuum "+" and "-" beam total power measurements is tagged with the actual antenna encoder positions. As a result, antenna tracking errors caused by wind gusts, for example, are actually recorded and taken into account in the data analysis stage.

For spectral line OTF, the same calibration and off measurements were used to calibrate all of the on measurements in the scanning rows until another off measurement was taken. Each total power off measurement was given its own scan number. All of the spectra or continuum "+" and "-" beam total power measurements in each scanning row were concatenated along with arrays of time and position information and stored on disk under a single scan number with a single header. The header information for each scanning row contained the scan number of the previous off (for spectral line OTF) and calibration measurements.

Given the scanning parameters, the positioning system was configured for tracking rates, row offsets, and the duration of a scanning row. The spectral line data taking backend instructed the tracking system to move to position and begin scanning. Using some handshaking bits on the telescope's status and monitor (SAM) bus, the two systems were synchronized at the start of each row. In addition, both the tracking and backend computers had IRIG clock cards that were driven by the observatory GPS standard clock. The data backend read out the spectrometer system every $100 \mathrm{~ms}$ for spectral line OTF. The continuum data system was read out every $250 \mathrm{~ms}$. For both spectral line and continuum OTF, the data backend tagged each data parcel with the UT time stamp. For the digital autocorrelation spectrometer at the 12 Meter Telescope (called the Millimeter Autocorrelator (MAC)), before the data could be read out Fourier transforms of the $100 \mathrm{~ms}$ data samples needed to be calculated. To make data analysis as fast as possible, the FFT's were performed in realtime by the MAC control computers. Every $10 \mathrm{~ms}$, the tracking computer recorded its $\mathrm{Az} / \mathrm{El}$ position with respect to the field center. Finally, at the end of the scanning row, the position information from the tracking system was merged with the data. An interpolation of the position information was then made to align slight differences between the time stamps of the two data sets.

\section{AIPS processing}

AIPS (Greisen 2003) was the default analysis software used to process OTF data acquired with the NRAO $12 \mathrm{~m}$ Telescope (see Mangum 1999). The main processing task was SDGRD (developed by Eric Greisen), which read the raw $12 \mathrm{~m}$ ("sdd") data and gridded it using a variety of user-selectable convolution functions. Figure 13 shows a sample of an OTF image obtained with the NRAO 12 Meter Telescope and processed with the AIPS OTF image analysis tasks.

\section{Conclusion}

The On-The-Fly (OTF) imaging technique is an efficient singledish radio telescope imaging mode that has been implemented 


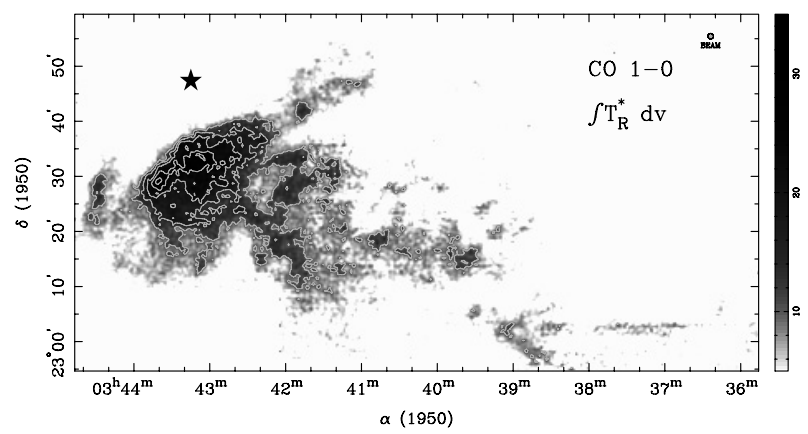

Fig. 13. CO $1 \rightarrow 0$ integrated intensity image of a molecular cloud near the Pleiades cluster. The Pleiades member Merope is located in the upper left portion of this image, just off of the main emission component. The $\mathrm{CO}$ emission appears to be tracing an interaction between the young stars in the Pleiades cluster and a nearby molecular cloud.

at several radio telescope observatories. The relative gain in observing efficiency is most extreme for the "classic" radio telescopes still in operation. This was certainly true for the NRAO 12 Meter Telescope. The NRAO 12 Meter Telescope began life as the 36 Foot Telescope, the telescope responsible for the birth of millimeter-wavelength molecular astronomy. Its history is one of success, innovation, and an unparalleled desire by the staff operating this facility over its 32 -year lifetime to provide a high level of service to the astronomical community.

The 36 Foot Telescope made its first millimeter-wavelength astronomical measurements in October 1968. This was the start of a period of explosive growth in this new area of astronomical research, during which most of the dozens of molecular species known to exist in the interstellar medium were first detected with the 36 Foot.

In the early 1980s the telescope's reflecting surface and surface support structure were replaced and in 1984 it was rechristened as the 12 Meter Telescope. Its scientific program subsequently evolved from one dominated by astrochemistry to a broader mix of studies of molecular clouds and Galactic star formation, evolved stars, astrochemistry, and external galaxies.

The NRAO 12 Meter Telescope was the only millimeterwavelength telescope in the US operated full-time as a national facility. More than 150 visitors used it each year. It offered users flexibility and the opportunity to respond quickly to new scientific developments. Its low-noise receiving systems covered a wide range of frequencies - all atmospheric windows from $68 \mathrm{GHz}$ to $300 \mathrm{GHz}$ - and much attention was given to making the instrument work reliably throughout this range. Flexible spectral line and continuum back-ends allowed the observer to match the instrument to the scientific goals. The development of multi-beam receivers and the on-the-fly observing technique inaugurated a new era of high-speed source mapping on angular scales complementary to those of the millimeter-wave interferometers. The telescope control system offered great flexibility and provided a proven remote observing capability.

On February 22, 2000, NRAO announced that it would close the 12 Meter Telescope at the end of the current observing season. On 26 July 2000, the NRAO 12 Meter Telescope made its final astronomical measurements as a US national astronomical facility. The telescope however continues to be operated as part of the University of Arizona's Steward Observatory. The final NRAO data set was an on-the-fly image of the CO $1 \rightarrow 0$ emission from a star formation region in the Cygnus- $\mathrm{X}$ region.

\section{References}

Ball, J. A. 1976, in Meth. Exper. Phys., Vol. 12, Part C,

Astrophysics: Radio Observations, Chap. 4.3, ed. M. L. Meeks

Emerson, D. T., Klein, U., \& Haslam, C. G. T. 1979, A\&A, 76, 92

Emerson, D. T. 1996, Increasing the Yield of Our Telescopes, in CO: TwentyFive Years of Millimeter-Wave Spectroscopy, Proc. 170th Symposium of the International Astronomical Union held in Tucson, Arizona, May 29-June 5, 1995, ed. W. B. Latter, S. J. E. Radford, P. R. Jewell, J. G. Mangum, \& J. Bally (Kluwer Academic Publishers), 207

Greisen, E. W. 1998, AIPS CookBook, NRAO. Socorro, NM

Greisen, E. W. 2003, Information Handling in Astronomy - Historical Vistas, ed. A. Heck (Dordrecht: Kluwer Academic Publishers), Astrophysics and Space Science Library, 285, 109

Haslam, C. G. T., Quigley, M. J. S., \& Salter, C. J. 1970, MNRAS, 147, 405

Jansky, K. G. 1932, Directional Studies of Atmospherics at High Frequencies, Proc. Inst. Radio Eng., 20, 1920

Mangum, J. G. 1999, On the Fly Observing at the 12 m, NRAO $12 \mathrm{~m}$ observer documentation

Mangum, J. G., Emerson, D. T., \& Greisen, E. 2000, in Imaging at Radio Through Submillimeter Wavel., ed. J. G. Mangum, \& S. J. E. Radford, ASP Conf. Ser., 217, 179

Schwab, F. R. 1980, VLA Scientific Memorandum \#132, NRAO, Socorro, NM Sullivan, W. T., III, 1978, Sky and Telescope, 56, 101, A New Look at Karl Jansky's Original Data 